\title{
Risk Factors Associated with Fall Awareness, Falls, and Quality of Life Among Ethnic Minority Older Adults in Upper Northern Thailand
}

\author{
Supakan Kantow' \\ Katekaew Seangpraw (iD) \\ Parichat Ong-artborirak ${ }^{2}$ \\ Prakasit Tonchoy' \\ Nisarat Auttama' \\ Sasivimol Bootsikeaw' \\ Monchanok Choowanthanapakorn' \\ 'School of Public Health, University of \\ Phayao, Phayao, 56000, Thailand; ' 2 Faculty \\ of Public Health, Chiang Mai University, \\ Chiang Mai, 50200, Thailand
}

Background: Falls are a major public health issue and one of the leading causes of morbidity and mortality among the older adults in many countries. Falls and their consequences have an impact on people's quality of life, particularly the older adults. However, there have been very few studies on falls among ethnic minority groups living in rural areas. The aim of this study was to examine factors related to fall awareness, falls, and quality of life among ethnic minority older adults living in northern Thailand.

Methods: A cross-sectional study was conducted among 462 older adults from ethnic minority groups living in rural communities in Phayao and Lamphun provinces of northern Thailand using a multi-stage sampling technique. The data were collected through face-toface interviews, with structured questionnaires covering health information, fall awareness, and quality of life (WHOQOL-OLD). The factors were determined using simple linear regression and binary logistic regression.

Results: The mean age of the participants was 68.2, Karen (35.5\%), Mien $(24.2 \%)$, indigenous (20.8\%), and Hmong (19.5\%). In the previous 12 months, 20.6\% of elders had experienced a fall, and $4.5 \%$ of them had experienced multiple falls. More than half of the elders had moderate fall awareness and quality of life (71.6\% and $68.6 \%$, respectively). Univariate analysis revealed that minority group, sociodemographic characteristics, sleep duration, and fear of falling were all found to be associated with fall awareness, falls, and quality of life score $(\mathrm{p}<0.001)$. There was a significant relationship among fall awareness, falls, and quality of life score $(\mathrm{p}<0.001)$. Compared to those who had not fallen in the previous year, the quality of life of the older adults who had fallen was about 11 points lower. Conclusion: Several independent variables were discovered to be fall risk factors and quality of life indicators. It is critical to recognize the risk factors to promote fall prevention programs that are appropriate for the older adults among ethnic minorities. Therefore, public health agencies and local government should be aware of the problem and initiate an implementation program and policy to prevent falls and improve the quality of life among ethnic minority older adults.

Keywords: fall awareness, falls, quality of life, older adults, ethnic minority

\section{Introduction}

Falls among the older adults have become a major public health problem in many countries. ${ }^{1}$ According to the World Health Organization, nearly 424,000 fatal falls occur each year (an average of 1160 people per day). ${ }^{1,2}$ Falls have become a common challenge in the public health domain because they lead to physical injury, disability, and mortality, ${ }^{3}$ and affect quality of life among older adults. ${ }^{1,2,4}$ Previous studies have
Correspondence: Katekaew Seangpraw School of Public Health, University of Phayao, Phayao, 56000, Thailand Tel +6654466 66

Email eungkaew@gmail.com 
shown that the majority of falls occur among older adults aged 60 years and over; and the incidence of falls increases with age. ${ }^{5}$ Approximately one-third of older adults aged 65 years and over living in the community experiences at least one fall each year; and this proportion exceeds $40 \%$ among those aged 85 years and older. ${ }^{4,6}$ In addition, studies have found that approximately $30-60 \%$ of falls take place among older adults who live in a rural community. ${ }^{7}$ While many socio-demographic characteristics contribute to the quality of life of the older adults, such as age, socio-economic status, and marital status, the extant literature has paid particular attention to possible risk factors for falls. ${ }^{5}$ These factors include age, number of chronic diseases, body composition, muscle strength, functional mobility, and performance measures related to balance. ${ }^{3}$ In addition, falls and adverse health effects impact individual's' costs, health care system, and funding by the government. ${ }^{2,3}$ Self-awareness is a model consisting of three hierarchical levels: cognitive (intellectual), emergence, and anticipation. ${ }^{8}$ Other studies have found that education is related to self-awareness and is statistically significant for fall prevention behaviors. ${ }^{9}$ As a result, self-awareness may be an important factor to consider when analyzing fall risks. ${ }^{9}$

Thailand is currently facing the challenge of a rapidly aging population. ${ }^{10}$ According to the Thailand National Statistical Office, older adults constitute $17.5 \%$ of the population. ${ }^{10}$ Thailand's northern region has the highest proportion of older adults (19.8\%). ${ }^{10}$ Lamphun and Phayao, in particular, are two of seven provinces in the northern region with the highest number of older adults $(24.0 \%$ and $19.0 \%) .{ }^{10,11}$ According to a survey, there are one-and-a-half times more older adult females than males in the northern region. ${ }^{11}$ Most of the fall cases among older adult females took place around the home or in fenced areas $(55 \%){ }^{11}$ Sixty percent of male fall cases took place while traveling or working outside of the home. ${ }^{11}$ Previous studies have found that older adults who fall frequently experience physical injuries and a decline in their physical and mental health, leading to fear and a loss of confidence in performing daily activities. ${ }^{12,13}$ Some studies suggest that an individual's awareness, positive attitude, and health concerns are important factors in preventing falls. ${ }^{13,14}$ Quality of life is a term used in many fields and has a wide range of contexts depending on utility, health status, lifestyle, and satisfaction, ${ }^{15}$ in addition, quality of life is related to the perception individuals have of their life expectancy in the context of the community and culture in which they live. ${ }^{15-17}$ However, studies show that older Thai people are experiencing a deteriorating quality of life due to numerous factors, including regular alcohol consumption, lack of exercise, chronic health conditions, ${ }^{18}$ and a poor social environment; in addition, falling is one of the factors that impacts health and quality of life among elders. ${ }^{16,18}$ Falling affects older adults physically and mentally, and can lead to stress, financial dependence, decreased quality of life, the need for long-term care, and increased medical expenses. ${ }^{13}$ According to one study, financial independence is significantly associated with quality of life among older adults. $^{19}$

In the past, hill tribes, lowland, and indigenous people were ethnic minority groups who migrated from China, Myanmar, and Laos and came to settle in the northern region of Thailand. ${ }^{20,21}$ Ethnic minorities settled in all the provinces of northern Thailand, including Chiang Mai, Chiang Rai, Phayao, Lamphun, Nan, and Tak. ${ }^{20,21}$ The World Health Organization has reported that about four million ethnically diverse tribal hill people and highland settlers inhabit Thailand. ${ }^{22}$ According to an ethnic groups report, there are four major types of settlement characteristics: 1) Highland or hill tribe settlers, 2) Lowland settlers, 3) The Chao Lay group, and 4) Forest settlers. ${ }^{21,23}$ These settlers have their own culture, language, and way of life, especially regarding their beliefs in healthcare and health seeking behaviors. ${ }^{24}$ Their wooden houses are located in the mountains, with high stairs leading to distant outside bathrooms. ${ }^{25}$ Their ways of life are unique, depending on each the group to which they belong. ${ }^{25}$ Some studies have reported that the living environment is significantly associated with falls among older adults. ${ }^{18,26}$ This researcher has done extensive research on falls and the associated risk factors among ethnic older adults living in northern Thailand; however, this study found that only a few studies have focused on quality of life and falls among Thai older adults from ethnic minority groups. The main barriers to conducting research include inconvenient access to remote area, and the way of life, and beliefs among ethnic minority groups. ${ }^{11,24}$ Some studies have used a variety of fall assessment tools. ${ }^{12,13}$ These tools may be limited as they do not cover external factors that result in falls. ${ }^{13,25}$ Therefore, this study focuses on fall awareness, falls, quality of life, and risk factors associated with falls among older adults from ethnic minority groups, including highlanders, hill-tribe settlers (Mien and Hmong), and lowlanders (Karens and Indigenous people). In addition, the data presented will help healthcare practitioners and policy makers plan the implementation of fall prevention programs to improve the quality of life among 
older adults from ethnic minority groups living in the rural northern region of Thailand.

\section{Methodology}

This was a cross-sectional study based on the main project called "Unit of Excellence: Health Promotion and Quality of Life" and a health survey on older adults from ethnic minority groups living in northern Thailand. The quantitative data were collected in the rural areas of two provinces, Phayao and Lamphun, which are located in northern Thailand far from the urban centers. The area covered comprises large forest areas with high mountains, which makes it difficult for the locals to commute to urban centers. Accessing the study area was one of the challenges of this study it takes about 4-5 hours to reach the areas. Furthermore, the nearest primary and secondary healthcare units provided by the government are difficult to access. The data were obtained between February and May 2021.

A multi-stage sampling technique was designed to obtain the desired samples as follows Figure 1: a) Select provinces with a large population of ethnic minority groups, including the highlanders and hill tribe settlers

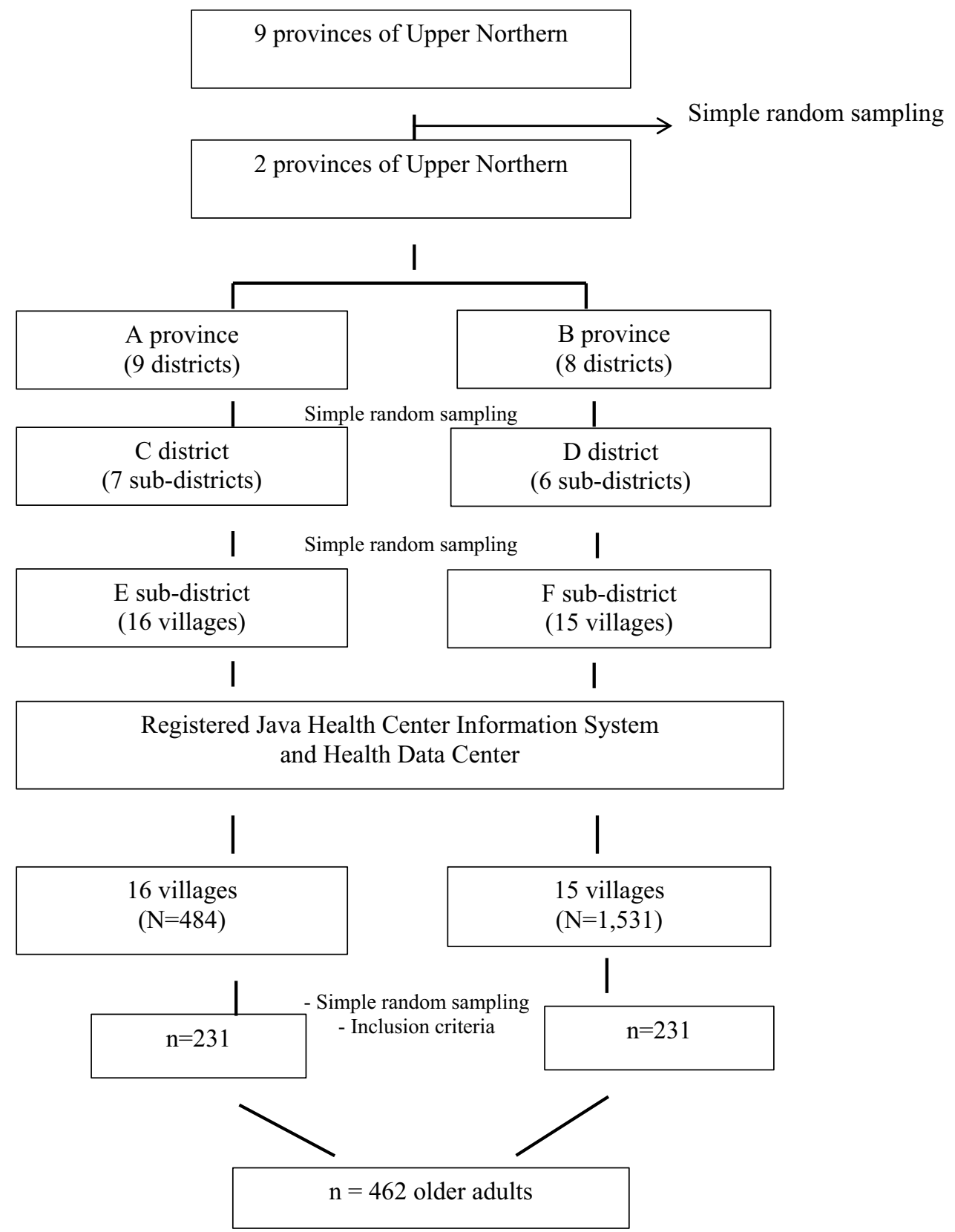

Figure I The flow of participants at enrollment in the project. 
(Mien and Hmong) and the lowlanders (Karens and Indigenous people). b) The recruitment process to select the samples was done based on a list of senior citizens enrolled in the 2018-2020 Health Data Center program and included Primary Care Units of the Java Health Center Information System (JHCIS). The samples were recruited using a simple random sampling technique. The inclusion criteria for the sample included: (1) Females and males aged 60 years and over; (2) older adults from ethnic minority groups who had registered with the High Area Health Development Center (Marginalized Ethnic Groups) Department of Health, Ministry of Public Health, (3) Resided in the area for at least two years, (4) Able to communicate in the local language, (5) Were not diagnosed as psychiatric patients, and (6) Willingness to participate in the study as evidenced by informed consent. Persons with cognitive impairment were excluded from the study. Sample size calculation was based on Cochran's formula $\left[Z^{2} \mathrm{pq} / \mathrm{e}^{2}\right.$, with a $95 \%$ confidence level $(\mathrm{Z}=1.96)$, a proportion of $50 \%$ for assuming maximum variability $(\mathrm{p}=0.5, \mathrm{q}=0.5)$, and $\pm 5 \%$ precision $(\mathrm{e}=0.05)] .^{27}$ To avoid losing incomplete data, the researcher included a $20 \%$ dropout rate among the participants. A total of 462 older adults were enrolled in the study.

Prior to conducting the research, 10 research assistants were recruited from each selected sub-district from the field of public health. These assistants included three public health scholars and seven village health volunteers. All the assistants were able to communicate in the local northern and ethnic languages; moreover, they had to have adequate knowledge about the culture, beliefs, and way of life of the ethnic minority groups. A four-hour workshop was organized in each area to clarify the objectives of the research, the data collection process and techniques, the scheduling of appointments, and the rights and privacy of the research participants. The workshops also ensured that all the research assistants had a common understanding of the same processes. The researcher translated the research material from a formal language to a local language. The research assistants were trained to enhance their communication skills to better communicate and understand the language, as well as the cultural and social environmental contexts of the samples being studied. This research process was conducted during the second wave of the COVID-19 epidemic in Thailand; therefore, all the research actions had to be authorized by the local government and community leaders. Furthermore, the researcher and the team had to follow preventive measures and update news and information from announcements by the government. Data collection was obtained using face-toface interviews, door- to- door techniques, and by interviewing participants waiting for health services at a health center. The interviews were performed between 9:00 am and $-5: 00 \mathrm{pm}$ or at a convenient time. The duration of the interviews was approximately 30-45 minutes.

The questionnaires used in the study were applied from literature reviews and related research ${ }^{13,16,18}$ in order to be appropriate and suitable for the rural community context. The questionnaires were divided into four sections: general information, health information, fall awareness, and quality of life. The general information included gender, age, marital status, education, and income efficiency. The health information included alcohol consumption, smoking, exercise, BMI, blood pressure (measured at the time of the interview by a research assistant), current disease, sleep, fear of falling, falls over a period of a year, and fall injuries sustained. The questionnaire on awareness toward fall risks among older adults which was modified from existing research, ${ }^{13}$ consisted of 18 questions using a Likert scale with five choice options, ranging from strongly disagree to strongly agree. Examples of questions are, "As you get older, you will increase your own caregiving to avoid falling"; "You will either organize electrical cables that clutter walkways to reduce the risk of stumbling or ask your caregiver to do so"; "You pay attention when choosing shoes that are suitable for you"; or "You will be careful when walking on rough or uneven surfaces". The rating scores are classified as follows: $72-$ $90=$ good, $54-71=$ moderate, and $18-53=$ low. The questionnaire on quality of life was developed by the World Health Organization (WHOQOL-OLD) ${ }^{16,28}$ and; translated into the Thai version. ${ }^{18}$ The questionnaire consisted of 24 items in six domains, including 1) Sensory functioning (SAB), 2) Autonomy (AUT), 3) Past present, and future activities (PPE), 4) Social participation (SOP), 5) Death and dying (DAD), and 6) Intimacy (INT). The questionnaire used a rating scale ranging from 1 (not at all) to 5 (an extreme amount). Possible scores in each domain ranged from 4 to 20 points.

The score is totaled at three levels of quality of life: low (scores between 24-55), moderate (scores between 56-88), and high (scores between 89-120). The questionnaire on fall awareness was checked by three experts in the field of health promotion, health behaviors, and the aging population. The researcher performed a pilot test using the questionnaires on similar characteristics of 30 samples. 
The Cronbach's alpha coefficients for the questionnaires on fall awareness and quality of life were 0.90 and 0.89 , respectively.

\section{Statistical Analysis}

The SPSS Version 17 software (SPSS Inc., Chicago, IL, USA), licensed from Chiang Mai University, was used to analyze the data. Descriptive statistics such as frequency, percentage, minimum (Min), maximum, (Max), mean with standard deviation (Mean $\pm \mathrm{SD}$ ) were presented for the characteristics of the sample and to assess variable distribution. The variables related to fall awareness and quality of life were determined using simple linear regression. To find factors linked to falls in the previous year among the ethnic older adults, binary logistic regression was used and odds ratio (OR) with 95\% confidence interval (CI) was reported. The significance level for statistical tests was set at a p-value of less than 0.05 .

\section{Results}

A total of 462 older adults (females (51.7\%), males $(48.2 \%))$ people completed the questionnaires. The average age was $68.2(\mathrm{SD}=6.9)$ and ages ranged from 60 to 104 years. Among the study group from the highlands were Mien (24.2\%) and, Hmong (19.5\%), and the group from the lowlands comprised Karen (35.5\%) and Indigenous (20.8\%). Overall, almost half of the older adults $(48.8 \%)$ had a normal BMI. The following, results were also achieved: systolic blood pressure (SBP) between 130/139 mmHg (35.5\%), diastolic blood pressure (DBP) between $80-89 \mathrm{mmHg}(50.0 \%)$. In terms of health, almost half of the older adults $(42.6 \%)$ had a current disease, such as hypertension (15.8\%), hyperlipidemia (10.8\%), diabetes mellitus $(4.5 \%)$, stroke $(4.5 \%)$, depression $(2.4 \%)$, tuberculosis $(2.2 \%)$, kidney disease $(1.9 \%)$, or orthopedic disease $(0.4 \%)$. More characteristics of older adults are presented in Table 1.

Table 2 summarizes the level of awareness toward fall risks, falls, and quality of life among older adults in minority groups. The scores of fall awareness ranged from 50-83, with a mean of $63.8(\mathrm{SD}=8.4)$. About half of the older adults (71.6\%) had a moderate level of fall awareness followed by a high (17.1\%), and low (11.3\%) awareness level. About $25 \%$ of the older adults in the study had fallen at least once in the previous year, with $19.9 \%$ of the older adults suffering injuries as a result of the fall. Quality of life among the older adults ranged from 44-97, with the mean equal to 65.6 $(\mathrm{SD}=10.6)$. The findings show that the majority of older adults $(68.6 \%)$ had a moderate level of quality of life, followed by a low level (24.9\%); and only a few of them had a high quality of life $(6.5 \%)$.

Univariate analysis of linear regression revealed that minority group, age, marital status, education, financial status, exercise, BMI, high blood pressure, having a disease, sleep duration, and fear of falling were all found to be associated with scores of awareness toward fall risks among the older adults $(\mathrm{p}<0.05)$ (Table 3$)$. Factors associated with falls are shown in Table 4. Logistic regression analysis showed that all the factors under investigation, except for marital status and smoking, were linked to falls $(p<0.05)$. The modeled odds of falls, per unit increase in fall awareness, decreased by $26 \%(\mathrm{OR}=0.74,95 \% \mathrm{CI}=0.70-0.78)$. In addition, fall awareness accounts for $57.7 \%$ of the variance in the log odds of one year falls. Besides, the results of simple linear regression show that minority group, gender, financial status, high blood pressure, having a disease, sleep duration, and fear of falling were related to quality of life among the older adults $(\mathrm{p}<0.05)$ (Table 5). There was a low positive correlation between fall awareness and quality of life, with a standardized coefficient of $0.36(\mathrm{p}<0.001)$. Compared to those who had no history of falls in the previous year, the quality of life of the older adults who had fallen was about 11 points lower. The single factors of fall awareness and falls can explain $12.7 \%$ and $20.0 \%$ of the variance in the quality of life among older adults of various ethnicities, respectively.

\section{Discussion}

There was a significant relationship among personal factors, fall awareness, falls, and quality of life in older adults aged 60 years and over from ethnic minority groups living in the rural areas of northern Thailand. Most older adults had moderate and low levels of fall awareness. This can be explained by the fact that nearly $80 \%$ of the older adults do not have a formal education, which could indicate a lack of knowledge and understanding about health and prevention and a consequent lack of self-awareness. People who are more self-aware haves a better attitude and self-efficacy, which enables them to prevent disease and engage in good health practices. ${ }^{29}$ A person's perception affects their attitudes, awareness, and self-efficacy, which leads to an understanding of the importance of good health. ${ }^{29}$ Accordingly, self-awareness has an effect on fall prevention, which suggests that a program aimed at raising fall awareness among senior ethnics should be considered. $^{30}$ 
Table I Sociodemographic of Elderly Categorized Based on Minority Groups

\begin{tabular}{|c|c|c|c|c|c|}
\hline \multirow[t]{2}{*}{ Variables } & Overall $(n=462)$ & Karen $(n=164)$ & Mien $(n=|| 2)$ & Indigenous $(\mathrm{n}=96)$ & Hmong $(n=90)$ \\
\hline & $n(\%)$ & $n(\%)$ & $n(\%)$ & $n(\%)$ & $n(\%)$ \\
\hline \multicolumn{6}{|l|}{ Gender } \\
\hline Male & $223(48.3)$ & $90(54.9)$ & $51(45.5)$ & $38(39.6)$ & $44(48.9)$ \\
\hline Female & $239(51.7)$ & $74(45.1)$ & $61(54.5)$ & $58(60.4)$ & $46(51.1)$ \\
\hline \multicolumn{6}{|l|}{ Age (years) } \\
\hline Mean \pm SD & $68.2 \pm 6.9$ & $71.5 \pm 7.3$ & $67.3 \pm 5.0$ & $62.2 \pm 1.8$ & $69.8 \pm 7.3$ \\
\hline Min-Max & $60-104$ & $60-104$ & $60-82$ & $60-67$ & $60-98$ \\
\hline \multicolumn{6}{|l|}{ Marital status } \\
\hline Single/widowed/separated & $55(11.9)$ & $37(22.6)$ & $2(1.8)$ & $13(13.5)$ & $3(3.3)$ \\
\hline Married & $407(88.1)$ & $127(77.4)$ & $110(98.2)$ & $83(86.5)$ & $87(96.7)$ \\
\hline \multicolumn{6}{|l|}{ Education } \\
\hline No & $364(78.8)$ & $139(84.8)$ & $95(84.8)$ & $58(60.4)$ & $72(80.0)$ \\
\hline Yes & $98(21.2)$ & $25(15.2)$ & $17(15.2)$ & $38(39.6)$ & $18(20.0)$ \\
\hline \multicolumn{6}{|l|}{ Financial status } \\
\hline Insufficient & $305(66.0)$ & $91(55.5)$ & $87(77.7)$ & $56(58.3)$ & $71(78.9)$ \\
\hline Sufficient & $157(34.0)$ & $73(44.5)$ & $25(22.3)$ & $40(41.7)$ & $19(21.1)$ \\
\hline \multicolumn{6}{|l|}{ Alcohol consumption } \\
\hline No & $302(65.4)$ & $112(68.3)$ & $74(66.1)$ & $59(61.5$ & $57(63.3)$ \\
\hline Yes & $160(34.6)$ & $52(31.7)$ & $38(33.9)$ & $37(38.5)$ & $33(36.7)$ \\
\hline \multicolumn{6}{|l|}{ Smoking status } \\
\hline Non-smoker & $357(77.3)$ & $127(77.4)$ & $89(79.5)$ & 74(77.1) & $67(74.4)$ \\
\hline Smoker & $105(22.7)$ & $37(22.6)$ & $23(20.5)$ & $22(22.9)$ & $23(25.6)$ \\
\hline \multicolumn{6}{|l|}{ Types of physical activity } \\
\hline Does not activity & $339(73.4)$ & $123(75.0)$ & $82(73.2)$ & $63(65.6)$ & $71(78.9)$ \\
\hline Exercise & $123(26.6)$ & $4 I(25.0)$ & $30(26.8)$ & 33(34.4) & $19(21.1)$ \\
\hline \multicolumn{6}{|l|}{ Body Mass Index $\left(\mathrm{kg} / \mathrm{m}^{2}\right)$} \\
\hline Mean \pm SD & $23.1 \pm 3.3$ & $23.5 \pm 3.6$ & $22.6 \pm 2.9$ & $22.6 \pm 2.9$ & $23.5 \pm 3.3$ \\
\hline Min-Max & $15.8-37.8$ & $16.0-37.8$ & $16.0-32.0$ & $\mid 5.8-31.6$ & $16.9-32.4$ \\
\hline \multicolumn{6}{|l|}{ Systolic blood pressure (SBP) } \\
\hline$<140 \mathrm{mmHg}$ & $282(61.0)$ & $106(64.6)$ & $62(55.4)$ & $64(66.7)$ & $50(55.6)$ \\
\hline$\geq 140 \mathrm{mmHg}$ & $180(39.0)$ & $58(35.4)$ & $50(44.6)$ & $32(33.3)$ & $40(44.4)$ \\
\hline Mean \pm SD & $134.1 \pm 14.8$ & $133.3 \pm 14.9$ & $136.5 \pm 12.7$ & $131.3 \pm 15.8$ & $135.5 \pm 15.5$ \\
\hline Min-Max & $90-204$ & 90-204 & $100-16 \mid$ & $94-168$ & $100-166$ \\
\hline \multicolumn{6}{|l|}{ Diastolic blood pressure (DBP) } \\
\hline$<90 \mathrm{mmHg}$ & $369(79.9)$ & $130(79.3)$ & $91(81.2)$ & $83(86.5)$ & $65(72.2)$ \\
\hline$\geq 90 \mathrm{mmHg}$ & $93(20.1)$ & $34(20.7)$ & $21(18.8)$ & $13(13.5)$ & $25(27.8)$ \\
\hline Mean \pm SD & $83.1 \pm 8.2$ & $82.8 \pm 8.6$ & $84.3 \pm 6.3$ & $81.2 \pm 8.3$ & $83.9 \pm 9.0$ \\
\hline Min-Max & $56-116$ & $56-116$ & $67-100$ & $60-100$ & $64-106$ \\
\hline \multicolumn{6}{|l|}{ Current disease } \\
\hline No & $265(57.3)$ & $89(54.3)$ & $59(52.7)$ & $75(78.1)$ & $42(46.7)$ \\
\hline Yes & $197(42.6)$ & $75(45.7)$ & $53(47.3)$ & $21(21.9)$ & $48(53.3)$ \\
\hline \multicolumn{6}{|l|}{ Sleep duration } \\
\hline$<7 \mathrm{hrs}$ & $258(55.8)$ & $99(60.4)$ & $54(48.2)$ & $53(55.2)$ & $52(57.8)$ \\
\hline$\geq 7 \mathrm{hrs}$ & 204(44.2) & $65(93.6)$ & $58(51.8)$ & $43(44.8)$ & $38(42.2)$ \\
\hline \multicolumn{6}{|l|}{ Fear of falling } \\
\hline No & $257(55.6)$ & $84(51.2)$ & $66(58.9)$ & $61(63.5)$ & $46(51.1)$ \\
\hline Yes & 205(44.4) & $80(48.8)$ & $46(4 I .1)$ & $35(36.5)$ & $44(48.9)$ \\
\hline
\end{tabular}


Table 2 Descriptive Analysis of Fall Awareness, Falls, and Quality of Life Among Elderly

\begin{tabular}{|c|c|c|c|c|c|}
\hline \multirow[t]{2}{*}{ Variables } & Overall $(n=462)$ & Karen $(n=164)$ & Mien $(n=1 \mid 2)$ & Indigenous $(n=96)$ & Hmong $(n=90)$ \\
\hline & $n(\%)$ & $n(\%)$ & $n(\%)$ & $n(\%)$ & $n(\%)$ \\
\hline \multicolumn{6}{|l|}{ Fall awareness } \\
\hline Low level (scores $\leq 53$ ) & $52(11.3)$ & $\mathrm{II}(6.7)$ & $16(14.3)$ & $6(6.3)$ & $19(21.1)$ \\
\hline Moderate level (scores 54-7I) & $33 I(7 I .6)$ & $140(85.4)$ & $67(59.8)$ & $72(75.0)$ & $52(57.8)$ \\
\hline High level (scores $\geq 72$ ) & $79(\mid 7.1)$ & 13(7.9) & $29(25.9)$ & $18(18.8)$ & $19(21.1)$ \\
\hline Mean \pm SD & $63.8 \pm 8.4$ & $62.3 \pm 7.0$ & $64.9 \pm 9.5$ & $65.1 \pm 7.7$ & $63.8 \pm 9.4$ \\
\hline Min-Max & $50-83$ & $50-79$ & $50-83$ & $53-82$ & $50-81$ \\
\hline \multicolumn{6}{|l|}{ Fall episode (s) } \\
\hline No fall & $346(74.9)$ & $130(79.3)$ & $8 \mathrm{I}(72.3)$ & $79(82.3)$ & $56(62.2)$ \\
\hline Falls & $116(25.1)$ & $34(20.7)$ & $3 \mid(27.7)$ & $17(17.7)$ & $34(37.8)$ \\
\hline Single fall & $95(20.6)$ & $26(15.8)$ & $27(24.1)$ & I7(I7.7) & $25(27.8)$ \\
\hline Recurrent falls & $21(4.5)$ & $8(4.9)$ & $4(3.6)$ & $0(0.0)$ & $9(10.0)$ \\
\hline \multicolumn{6}{|l|}{ Falling injury } \\
\hline No & $370(80.1)$ & $132(80.5)$ & $92(82.1)$ & $82(85.4)$ & $64(7 I . I)$ \\
\hline Yes & $92(19.9)$ & $32(19.5)$ & $20(17.9)$ & $14(14.6)$ & $26(28.9)$ \\
\hline \multicolumn{6}{|l|}{ Quality of life } \\
\hline Low level (scores $\leq 55$ ) & $115(24.9)$ & $27(16.5)$ & $33(29.5)$ & $21(21.9)$ & $34(37.8)$ \\
\hline Moderate level (scores 56-88) & $317(68.6)$ & $128(81.1)$ & $68(60.7)$ & $65(67.7)$ & $5 I(56.7)$ \\
\hline High level (scores $\geq 89$ ) & $30(6.5)$ & $9(2.4)$ & $\mathrm{II}(9.8)$ & $10(10.4)$ & $5(5.6)$ \\
\hline Mean \pm SD & $65.6 \pm 10.6$ & $67.7 \pm 9.8$ & $63.9 \pm 10.8$ & $66.6 \pm 11.3$ & $62.7 \pm 9.9$ \\
\hline Min-Max & $44-97$ & $44-90$ & $44-91$ & 47-92 & $50-97$ \\
\hline \multicolumn{6}{|l|}{ Sensory Ability (SAB) } \\
\hline Mean \pm SD & $8.9 \pm 2.1$ & $8.9 \pm 2.1$ & $8.6 \pm 1.9$ & $9.5 \pm 2.4$ & $8.6 \pm 1.8$ \\
\hline Min-Max & $5-16$ & $5-16$ & $5-16$ & $5-16$ & $5-13$ \\
\hline \multicolumn{6}{|l|}{ Autonomy (AUT) } \\
\hline Mean \pm SD & $10.2 \pm 2.8$ & $10.2 \pm 2.7$ & $9.8 \pm 3.2$ & $10.7 \pm 2.1$ & $10.1 \pm 2.9$ \\
\hline Min-Max & $2-28$ & $6-16$ & $5-28$ & $6-16$ & $5-16$ \\
\hline \multicolumn{6}{|l|}{ Past, Present, Future Activity (PPF) } \\
\hline Mean \pm SD & $12.2 \pm 2.9$ & $12.2 \pm 2.4$ & $12.2 \pm 3.9$ & $11.7 \pm 2.7$ & $12.7 \pm 2.6$ \\
\hline Min-Max & $7-43$ & $8-18$ & $8-43$ & $7-19$ & $8-17$ \\
\hline \multicolumn{6}{|l|}{ Social Participation (SOP) } \\
\hline Mean \pm SD & $12.1 \pm 2.9$ & $12.4 \pm 2.7$ & $11.8 \pm 2.9$ & $11.7 \pm 3.2$ & $12.3 \pm 3.0$ \\
\hline Min-Max & $5-20$ & $8-18$ & $7-19$ & $5-20$ & $8-17$ \\
\hline \multicolumn{6}{|l|}{ Death and Dying (DAD) } \\
\hline Mean \pm SD & $7.8 \pm 2.5$ & $7.7 \pm 2.6$ & $8.5 \pm 2.6$ & $6.6 \pm 3.3$ & $8.6 \pm 2.7$ \\
\hline Min-Max & $4-20$ & $4-19$ & $4-20$ & $4-17$ & $4-20$ \\
\hline \multicolumn{6}{|l|}{ Intimacy (INT) } \\
\hline Mean \pm SD & $14.3 \pm 2.2$ & $14.5 \pm 2.1$ & $14.6 \pm 1.8$ & $13.3 \pm 2.5$ & $14.5 \pm 1.9$ \\
\hline Min-Max & $8-20$ & $9-20$ & $10-20$ & $9-20$ & $8-18$ \\
\hline
\end{tabular}

The findings revealed that $25.1 \%$ of the study's participants experienced a fall over one year, which is comparable to a study that estimated a $27 \%$ prevalence of falls in Thai older adults aged 60 and over. ${ }^{31}$ However, the highlander group, particularly the Hmong, had more falls
(37.8\%). In terms of quality of life, the study showed that the majority of older adults obtained scores at a moderate level, followed by low and high levels of scores. This is in accordance with previous findings that used the same tool to assess the quality of life of ethnic 
Table 3 Factors Associated with Fall Awareness Among Elderly by Simple Linear Regression

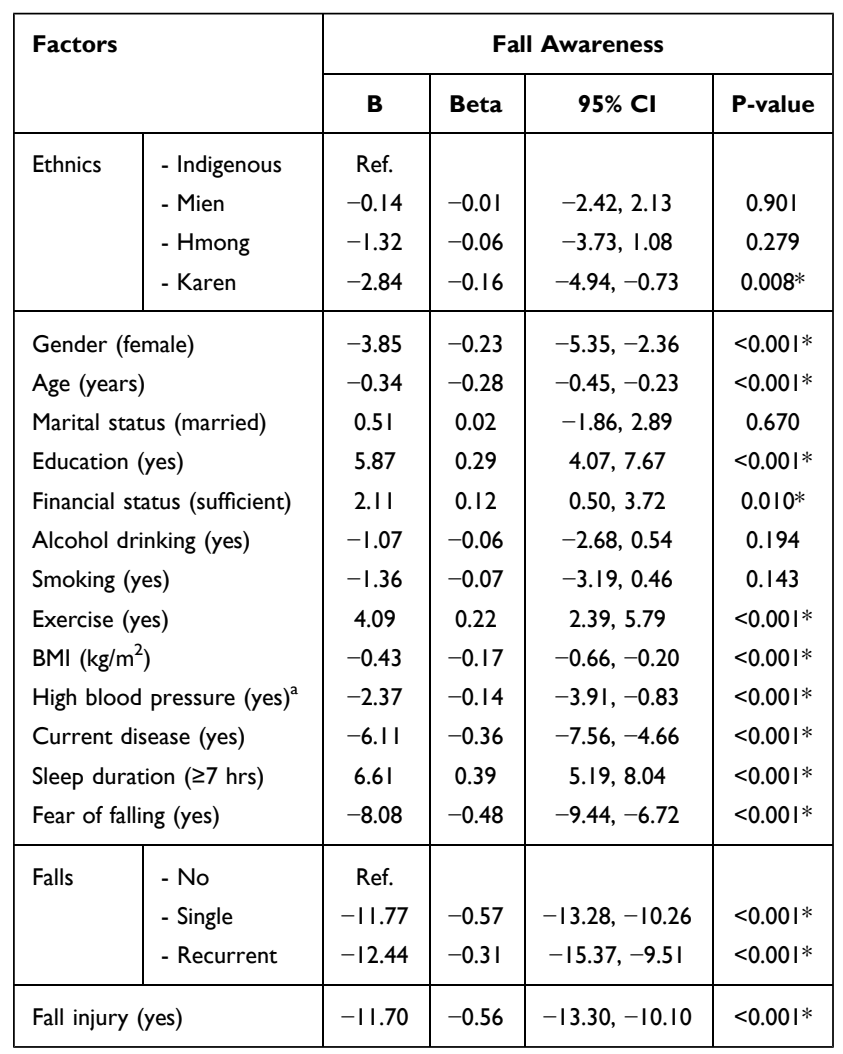

Notes: ${ }^{\text {a }}$ BP $\geq 140 \mathrm{mmHg}$ and/or DBP $\geq 90 \mathrm{mmHg}$. *Significant at the 0.05 level (2-tailed).

Abbreviations: B, regression coefficient; Beta, standardized coefficients; $\mathrm{Cl}$, confidence interval.

minority older adults in Thailand's rural communities. ${ }^{17}$ This is similar to the concept of self-perceived health, which is based on a person's experience and, learning, and is related to self-inflicted illness; it explained that as individual's age, their physical and cognitive behaviors would be declined. ${ }^{32}$ In the process, elders begin to rely on others for help in social and daily activities. ${ }^{33}$

Fall awareness is an important factor in this study. Demographic factors such as gender, age, education, and income adequacy were linked to fall awareness, a claim supported by previous studies, which found that fall awareness is associated with being male, ${ }^{34}$ and that there is an associations between the perception of falls with age and the level of education. ${ }^{35}$ The results of our study could indicate that a high background level has an impact on fall awareness. Our findings reveal that Karen ethnics had statistically lower fall awareness scores than indigenous ethnics, which may be the result of a higher education level among the indigenous group. Elders who had poor education could experience lower fall awareness,
Table 4 Factors Associated with I-Year Falls Among Elderly by Binary Logistic Regression

\begin{tabular}{|c|c|c|c|c|}
\hline \multicolumn{2}{|c|}{ Factors } & \multicolumn{3}{|c|}{ Falls } \\
\hline & & B & OR $(95 \% \mathrm{Cl})$ & P-value \\
\hline Ethnics & $\begin{array}{l}\text { - Indigenous } \\
\text { - Mien } \\
\text { - Hmong } \\
\text { - Karen }\end{array}$ & $\begin{array}{l}\text { Ref. } \\
0.58 \\
1.04 \\
0.19\end{array}$ & $\begin{array}{c}1 \\
1.78(0.91-3.47) \\
2.82(1.44-5.54) \\
1.22(0.64-2.32)\end{array}$ & $\begin{array}{c}0.007^{*} \\
0.091 \\
0.003^{*} \\
0.554\end{array}$ \\
\hline \multicolumn{2}{|c|}{ Gender (female) } & 1.34 & $3.83(2.39-6.13)$ & $<0.001 *$ \\
\hline \multicolumn{2}{|c|}{ Age (years) } & 0.07 & $1.07(1.04-1.11)$ & $<0.001 *$ \\
\hline \multicolumn{2}{|c|}{ Marital status (married) } & -0.02 & $0.98(0.51-1.87)$ & 0.950 \\
\hline \multicolumn{2}{|c|}{ Education (yes) } & -1.16 & $0.31(0.16-0.61)$ & $0.001 *$ \\
\hline \multicolumn{2}{|c|}{ Financial status (sufficient) } & -2.65 & $0.07(0.03-0.16)$ & $<0.001 *$ \\
\hline \multicolumn{2}{|c|}{ Alcohol drinking (yes) } & 0.82 & $2.28(\mid .48-3.5 I)$ & $<0.001 *$ \\
\hline \multicolumn{2}{|c|}{ Smoking (yes) } & 0.29 & $1.34(0.82-2.18)$ & 0.236 \\
\hline \multicolumn{2}{|c|}{ Exercise (yes) } & -2.24 & $0.11(0.05-0.25)$ & $<0.001 *$ \\
\hline \multicolumn{2}{|c|}{ BMI $\left(\mathrm{kg} / \mathrm{m}^{2}\right)$} & 0.12 & $1.13(1.06-1.20)$ & $<0.001 *$ \\
\hline \multicolumn{2}{|c|}{ High blood pressure (yes) } & -1.45 & $2.17(1.42-3.33)$ & $<0.001 *$ \\
\hline \multicolumn{2}{|c|}{ Current disease (yes) } & 2.11 & $8.24(5.01-13.56)$ & $<0.001 *$ \\
\hline \multicolumn{2}{|c|}{ Sleep duration ( $\geq 7 \mathrm{hrs}$ ) } & -3.65 & $0.03(0.01-0.07)$ & $<0.001 *$ \\
\hline \multicolumn{2}{|c|}{ Fear of falling (yes) } & 3.40 & $30.09(14.65-61.77)$ & $<0.001 *$ \\
\hline \multicolumn{2}{|c|}{ Fall awareness (scores) } & -0.30 & $0.74(0.70-0.78)$ & $<0.001 *$ \\
\hline
\end{tabular}

Notes: ${ }^{a} \mathrm{SBP} \geq 140 \mathrm{mmHg}$ and/or DBP $\geq 90 \mathrm{mmHg}$. *Significant at the 0.05 level (2-tailed).

Abbreviations: B, regression coefficient; OR, odds ratio; $\mathrm{Cl}$, confidence interval.

a decreased perception toward health, and an increased risk of falling. ${ }^{30}$ Additionally, health status including exercise, BMI, high blood pressure, current disease, sleep, and fear of falling were associated with fall awareness. Our findings are similar to a prior study showing participants with lower BMI and fewer comorbidities had higher falls awareness. ${ }^{34}$ Also, the idea that an awareness of one's own health conditions ${ }^{8}$ results in a person's increased awareness of their own health care aligns with Pender's theory. ${ }^{32}$ As a result, poor health condition may reflect poor fall awareness.

The risk factors of falls were among the key findings of this study, which established that Hmong highlanders had a higher risk of falls than indigenous lowlanders. This may be due to differences in the demographic characteristics of ethnic groups. In addition, demographic characteristics of Hmong elders include the fact that they live mostly in rural, highland, and mountainous areas; they have a unique culture, supernatural beliefs, live in natural surroundings, and travel into the forest; and their lifestyle and culture are different and unique from other ethnicities. ${ }^{24}$ According to the findings, women were more likely than men to have fallen in the previous year; getting older increased the risk of falling. That gender difference is 
Table 5 Factors Associated with Quality of Life Among Elderly by Simple Linear Regression

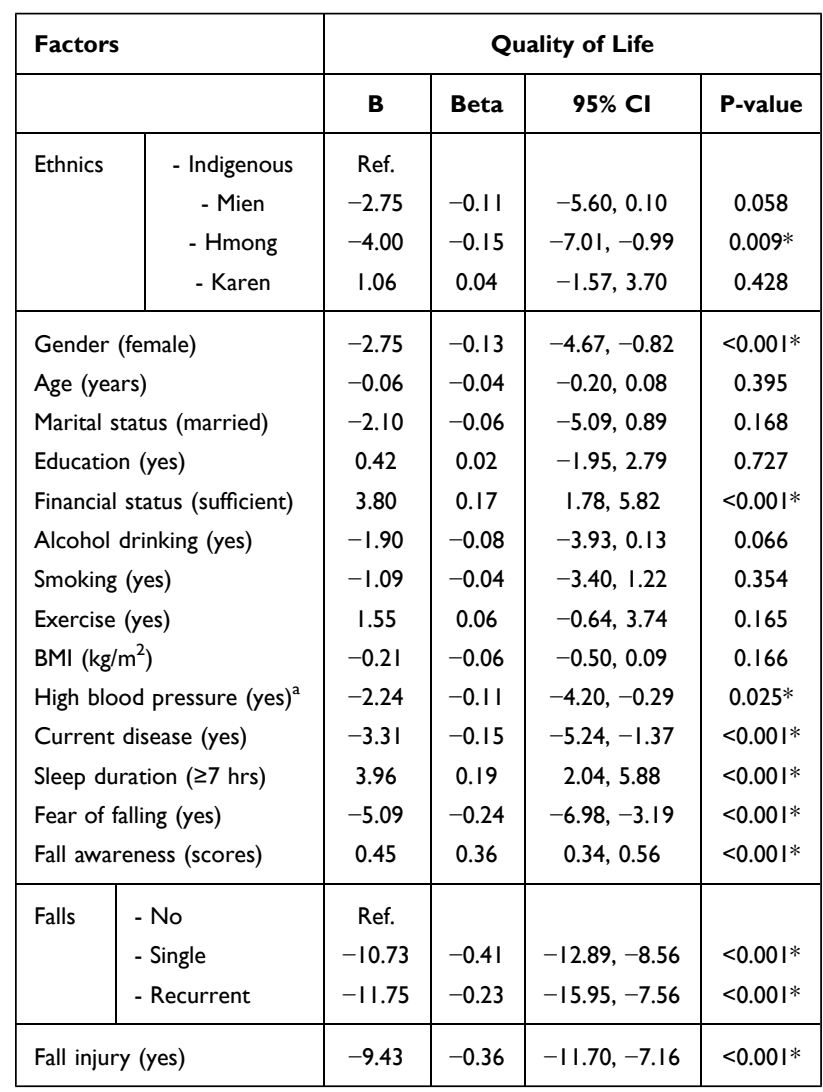

Notes: ${ }^{\mathrm{a} S B P} \geq 140 \mathrm{mmHg}$ and/or DBP $\geq 90 \mathrm{mmHg}$. *Significant at the 0.05 level (2-tailed).

Abbreviations: B, regression coefficient; Beta, standardized coefficients; Cl, confidence interval.

associated with physical structures and activities between men and women, such as muscle strength, bone density, and a body structure; that is one reason severe falls and injury are found more in females than in males. ${ }^{36}$ Previous studies have made similar findings: age and gender are among the risks factors of falls. ${ }^{11}$ Formal education is an important factors for preventing falls among older adults. According to prior studies, uneducated elders are twice as likely to experience falls as educated people..$^{22,37,38}$ Poor educational status and/or uneducated older adults may lack a proper understanding of the consequences of old age and, health related age and life adjustments. ${ }^{38}$ Therefore, dramatic changes in education and knowledge would improve the health of the older adults. ${ }^{39}$ In addition, our findings reveal a link between income and falls. This may be because insufficient income is one of the barriers to fall prevention and access to health care. ${ }^{40,41}$

Variables such as alcohol consumption, exercise, BMI, high blood pressure, current disease, and insufficient sleep were related to falls among ethnic older adults. Prior studies have found that individuals who regularly consume alcohol have a higher risk of falling than those who do not. ${ }^{42}$ Exercise deficiency and increasing BMI would increase the risk of falling among older adults. ${ }^{13,34}$ Excessive weight can result in a decrease in muscle strength and impair physical performance. ${ }^{2,34,43}$ Being overweight has a negative impact on a person's mobility and physical performance, contributing to an increased risk of falling and other related health risks, such as hypertension and cardiovascular disease. ${ }^{37,44}$ Also, the presence of chronic disease increases the burden and difficulty of physical mobility, resulting in an unsteady gait and imbalance, which increase the risk of falling. ${ }^{30,34} \mathrm{In}$ addition, insomnia a common symptom among older adults, could increase the risk of falling. ${ }^{13,44}$

Another important variable is a fear of falling, which was positively associated with falls. Fear of falling could create anxiety and stress, leading to an unsteady gait and imbalance. ${ }^{30,45}$ Elders who experience repeated falls may have an increased fear of falling. ${ }^{34,46}$ In terms of fall awareness, the study found that as one's score increased, the likelihood of falling decreased. Ethnic older adults who had experienced one or more falls in the previous year had about a 12-point lower fall awareness than those who had never fallen. This is in line with a previous study that found elders with a high level of fall awareness have fewer falls. ${ }^{30}$ As a result, several factors, particularly fall awareness, and play a role in the implementation of fallprevention programs.

This study found that, the Hmong had a lower quality of life than older adults from the indigenous lowland group. This is consistent with a previous study, which found that ethnic minority groups have a different quality of life. ${ }^{17}$ This could also be explained by a higher number of falling incidents among Hmong highlanders. As stated previously, ethnic groups have very unique ways of life, health beliefs, and social and environmental mores. This can be seen through their culture and the ways they dress, speak, and eat, their natural lifestyle, and their supernatural beliefs. ${ }^{17,24}$ These may have an impact on ethnic groups' quality of life.

The socio-demographic factors associated with quality of life in this study include gender, income sufficiency, blood pressure, current disease, and sleep. Older male adults have reported a higher quality of life than older female adults in low and middle income countries. ${ }^{47}$ Similarly, prior studies have found that income is positively associated with quality of life among older Thai 
people in rural areas. ${ }^{18}$ Having diseases such as hypertension and diabetes mellitus was strongly negatively associated with quality of life among the ethnic older adults. ${ }^{17}$ In addition, sleep deprivation has been linked to lower health-related quality of life in older community-dwelling people. ${ }^{48}$ Nevertheless, because this study was conducted during the COVID-19 pandemic, it is possible the pandemic had an impact on the study groups' employment, income, and access to health care, lowering their quality of life. ${ }^{10}$

This study found that fear of falling; fall awareness, falling, fall injuries, and quality of life are all closely linked among older adults from ethnic minority groups. Other studies have shown that individuals who are afraid of falling often experience poor quality of life. ${ }^{49,50}$ Fear of falling is recognized as an important psychological factor among older adults, who often develop paranoia and, experience reduced activity, and a loss of confidence in daily activities, as well as impaired physical, cognitive, and mental health functions, thus impeding their quality of life. ${ }^{50-52}$ Increased fall awareness may lead to fall prevention, resulting in better quality of life. In addition, our findings are consistent with many studies, and show an inverse relationship between falls and quality of life. ${ }^{6,53,54}$ Even falls that have resulted in minor injuries among older adults have been shown to exert a negative impact on their quality of life. ${ }^{3}$ It is important to point out the various factors affecting ethnic older adults' quality of life, which is one of the most satisfactory outcomes of all health policy. ${ }^{55}$

The strengths of this study included: 1) The participants were older adults from ethnic minority groups living in remote areas far from primary and secondary healthcare provided by the government. As far as we know, this was the first study to focus on ethnic Thai older adults in terms of fall awareness, falls, and quality of life. Our findings provide evidence to support fall-prevention programs and strategies for improving the quality of life among them; and 2) the participants were selected using a simple random sampling method, which helped reduce selection bias. There are several limitations in this study. First, a cross-sectional study makes it impossible to draw causal inferences. Second, the findings may not be applicable to older adults from other ethnic minority groups or those who live in other rural areas. Third, other factors, including access to health services, environmental and cultural factors, postural dispensations (standing, turning, walking, and changing positions), and their houses (high stairs leading to bathrooms outdoors) are important risk factors that were not included in this study. Accordingly, these variables could be used in future research. Finally, the respondents were asked about their fall experiences one year prior to the interview; therefore, we recommend a long-term study, such as a longitudinal prospective study to investigate the direction and cause of each component affecting quality of life.

\section{Conclusion}

This study found that several important factors are significantly associated with fall awareness, falls, and quality of life among older adults from ethnic minority groups. Managing these factors would be helpful in developing fall prevention programs to create an awareness of falls and their consequences and prevention, and to reduce the fear of falling. Moreover, such programs would empower older adults to be aware of health and well-being, which would enhance quality of life among them. The findings of this study can be used to initiate strategies for designing fall prevention programs suitable for the way of life, culture, and social environment context of older adults from ethnic minority groups living in rural communities. Therefore, public health agencies and local government should be aware of the problem and develop an implementation program and policy to prevent falls and improve the quality of life among ethnic minority older adults.

\section{Ethical Approval and Consent}

Ethical consideration was approved by the University of Phayao Human Ethics Committee, Thailand (No.1.3/020/ 63). Participants gave informed consent prior to data collection. This study was conducted in accordance with the Declaration of Helsinki, the Belmont Report, CIOMS Guideline, and International Conference on Harmonization in Good Clinical Practice (ICH-GCP).

\section{Acknowledgments}

The authors wish to acknowledge Health Promoting Hospitals of Health Promoting Hospitals in Mae-ta district Lamphun province and Pong district, Phayao province, Thailand, and all participants who voluntarily participated in this study.

\section{Funding}

This research project was supported by the Thailand science research and innovation fund and the University of Phayao the Unit of Excellence named "Health 
Promotion and Quality of Life" grant number FF64UoE009.

\section{Disclosure}

The authors report no conflicts of interest in this work.

\section{References}

1. World Health Organization. WHO Global Reports Falls Prevention in Older Age. 2007.

2. Wu H, Ouyang P. Fall prevalence, time trend and its related risk factors among elderly people in China. Arch Gerontol Geriatr. 2017;73:294-299. doi:10.1016/j.archger.2017.08.009

3. Ozcan A, Donat H, Gelecek N, Ozdirenc M, Karadibak D. The relationship between risk factors for falling and the quality of life in older adults. BMC Public Health. 2005;5(1):90. doi:10.1186/14712458-5-90

4. Kannus P, Sievanen H, Palvanen M, Jarvinen T, Parkkari J. Prevention of falls and consequent injuries in elderly people. Lancet. 2005;366(9500):1885-1893. doi:10.1016/S0140-6736(05) 67604-0

5. Zhao YL, Alderden J, Lind B, Stibrany J. Risk factors for falls in homebound community-dwelling older adults. Public Health Nurs. 2019;36(6):772-778. doi:10.1111/phn.12651

6. Chang NT, Chi LY, Yang NP, Chou P. The impact of falls and fear of falling on health-related quality of life in Taiwanese elderly. $J$ Community Health Nurs. 2010;27(2):84-95. doi:10.1080/ 07370011003704958

7. Cheng MH, Chang SF. Frailty as a risk factor for falls among community dwelling people: evidence from a meta-analysis. $J$ Nurs Scholarsh. 2017;49(5):529-536. doi:10.1111/jnu.12322

8. Crosson B, Barco PP, Velozo CA, et al. Awareness and compensation in postacute head injury rehabilitation. $J$ Head Trauma Rehabil. 1989;4(3):46-54. doi:10.1097/00001199-198909000-00008

9. Mihaljcic T, Haines TP, Ponsford JL, Stolwyk RJ. Self-awareness of falls risk among elderly patients: characterizing awareness deficits and exploring associated factors. Arch Phys Med Rehabil. 2015;96 (12):2145-2152. doi:10.1016/j.apmr.2015.08.414

10. Social situation and outlook. Thailand: National Statistical Office; 2021. Available from: https://www.dop.go.th/th/know/side/1/1/335. Accessed September 28, 2021.

11. Srichang N, Kavee L. Report of Falls of the Elderly (Age 60 Years and Over) in Thailand, 2017 - 2021 (Thai). Division of NonCommunicable Disease, Department of Disease Control, Ministry of Public Health; 2021.

12. Chittrakul J, Siviroj P, Sungkarat S, Sapbamrer R. Physical frailty and fall risk in community-dwelling older adults: a Cross-Sectional Study. J Aging Res. 2020;2020:3964973. doi:10.1155/2020/3964973

13. Chamnankit T, Ong-artborirak P, Wangrath J. Association between awareness of informal caregivers and falls in elderly patients with uncontrolled diabetes mellitus. Open Public Health J. 2020;13 (1):658-666. doi:10.2174/1874944502013010658

14. Ang SGM, O'Brien AP, Wilson A. Fall concern about older persons shifts to carers as changing health policy focuses on family, home-based care. Singapore Med J. 2018;59(1):9-11. doi:10.11622/ smedj.2018005

15. Dijkers M. Measuring quality of life: methodological issues. Am J Phys Med Rehabil. 1999;78(3):286-300. doi:10.1097/00002060199905000-00022

16. Power M, Kuyken W, Orley J. The World Health Organization Quality of Life Assessment (WHOQOL): development and general psychometric properties. Soc Sci Med. 1998;46(12):1569-1585. doi:10.1016/S0277-9536(98)00009-4
17. Seangpraw K, Ong-Artborirak P. Knowledge and behaviors toward health care for elderly patients with hypertension, and quality of life among informal caregivers in northern Thailand. $J$ Multidiscip Healthc. 2020;13:1771-1780. doi:10.2147/JMDH.S284886

18. Hongthong D, Somrongthong R, Ward P. Factors influencing the Quality of Life (Qol) among Thai older people in a rural area of Thailand. Iran J Public Health. 2015;44(4):479-485.

19. Shin KR, Byeon YS, Kang Y, Oak J. A study on physical symptom, activity of daily living, and health-related quality of life (HRQoL) in the community-dwelling older adults. Taehan Kanho Hakhoe Chi. 2008;38(3):437-444.

20. Princess Maha Chakri Siridhorn Anthropology Center. Hill tribe. Thai; 2017. Available from: http://www.sac.or.th/main/index.php. Accessed September 28, 2021.

21. Ong-Artborirak P, Seangpraw K. Association between self-care behaviors and quality of life among elderly minority groups on the border of Thailand. J Multidiscip Healthc. 2019;12:1049-1059. doi:10.2147/ JMDH.S227617

22. World Health Organization. Migrant and Vulnerable Health Program: WHO-Thailand Office Work-Plan 2017. 2016:2-6

23. Health Center of Ethnic Group, Migrant Peoples and Migrant Workers. Health promotion and environmental management of the Lua Hill Tribe people (Thai). Thailand: Department of Health Ministry of Public Health; 2015. Available from: https://hhdclam pang.anamai.moph.go.th/database/admin/research/file/ 200814102014.pdf. Accessed September 28, 2021.

24. Apidechkul T. Prevalence and factors associated with type 2 diabetes mellitus and hypertension among the hill tribe elderly populations in northern Thailand. BMC Public Health. 2018;18(1):694. doi:10.1186/ s12889-018-5607-2

25. Sirimuangmoon K, Saicharoent C. Beliefs and spatial organization of Karen houses in Prabaht-Huaytom Village, Nasai Sub-district, Li District, Lumphun Province. J Environ Design. 2016;3(1):113-142.

26. Coutinho ES, Fletcher A, Bloch KV, Rodrigues LC. Risk factors for falls with severe fracture in elderly people living in a middle-income country: a Case Control Study. BMC Geriatr. 2008;8(1):21. doi:10.1186/1471-2318-8-21

27. Cochran WG. Sampling Techniques. New York: John Wiley \& Sons. Inc. Book; 1977.

28. Power M, Quinn K, Schmidt S. Development of the WHOQOL-Old module. Qual Life Res. 2005;14(10):2197-2214. doi:10.1007/s11136005-7380-9

29. Coopersmith S. Self -Esteem Inventory. 2nd ed. California: Consulting Psychologist Press; 1981.

30. Chehuen Neto JA, Brum IV, Braga NA, et al. Fall awareness as a determining factor of this event among elderly community residents. Geriatr Gerontol Aging. 2017;11(1):25-31.

31. Srichang N, Kaawi L. Forecast of falls in the elderly (aged 60 and over) in Thailand, 2017-2021 (Thai). Bureau of Non Communicable Disease, Department of Disease Control, Ministry of Public Health; 2016. Available from: http://www.thaincd.com/2016/mission/documentsdetail.php?id=12095\&tid=\&gid=1-027. Accessed September 28, 2021.

32. Pender NJ. Health Promotion in Nursing Practice. 2nd ed. Stamford, CT: Appleton \& Lange; 1987.

33. Somrongthong R, Wongchalee S, Yodmai K, Kuhirunyaratn $P$, Sihapark S, Mureed S. Quality of life and health status among Thai elderly after economic crisis, Khon Kanen Province, Thailand. Eur J Sci Res. 2013;112(3):314-324.

34. Goh JW, Singh DKA, Mesbah N, Hanafi AAM, Azwan AF. Fall awareness behaviour and its associated factors among community dwelling older adults. BMC Geriatr. 2021;21(1):226. doi:10.1186/ s12877-021-02122-z

35. Gamage N, Rathnayake N, Alwis G. Knowledge and perception of falls among community dwelling elderly: a study from southern Sri Lanka. Curr Gerontol Geriatr Res. 2018;7653469. doi:10.1155/2018/ 7653469 . 
36. Stevens JA, Sogolow ED. Gender differences for non-fatal unintentional fall related injuries among older adults. Inj Prev. 2005;11 (2):115-119. doi:10.1136/ip.2004.005835

37. Stewart Williams J, Kowal P, Hestekin H, et al.; SAGE collaborators. Prevalence, risk factors and disability associated with fall-related injury in older adults in low- and middle-income countries: results from the WHO Study on global AGEing and adult health (SAGE). BMC Med. 2015;13(1):147. doi:10.1186/s12916-015-0390-8

38. Janakiraman B, Temesgen MH, Jember G, et al. Falls among community-dwelling older adults in Ethiopia; a Preliminary Cross-Sectional Study. PLoS One. 2019;14(9):e0221875. doi:10.1371/journal.pone.0221875

39. Kye B, Arenas E, Teruel G, Rubalcava L. Education, elderly health, and differential population aging in South Korea: a demographic approach. Demogr Res. 2014;30:753-794. doi:10.4054/ DemRes.2014.30.26

40. Lecktip C, Woratanarat T, Bhubhanil S, Lapmanee S. Risk factors for falls in elderly (Thai). $J$ Med Health Sci. 2019;26(1):85-103.

41. Sarapun A, Sirisopon N, Kainaka P, et al. Factors ralated to a fall prevention behaviors of elderly. J Royal Thai Army Nurs. 2017;18 (Supplement):215-222.

42. Zhang L, Ding Z, Qiu L, Li A. Falls and risk factors of falls for urban and rural community-dwelling older adults in China. BMC Geriatr. 2019;19(1):379. doi:10.1186/s12877-019-1391-9

43. Himes CL, Reynolds SL. Effect of obesity on falls, injury, and disability. J Am Geriatr Soc. 2012;60(1):124-129. doi:10.1111/ j.1532-5415.2011.03767.x

44. Brassington GS, King AC, Bliwise DL. Sleep problems as a risk factor for falls in a sample of community-dwelling adults aged 64-99 years. J Am Geriatr Soc. 2000;48(10):1234-1240. doi:10.1111/ j.1532-5415.2000.tb02596.x

45. Kendhapedi KK, Devasenapathy N. Prevalence and factors associated with frailty among community-dwelling older people in rural Thanjavur district of South India: a Cross-Sectional Study. BMJ Open. 2019;9(10):e032904. doi:10.1136/bmjopen-2019-032904

46. Endsley MR. Toward a theory of situation awareness in dynamic systems. Hum Factors. 1995;37(1):32-64. doi:10.1518/ 001872095779049543
47. Lee $\mathrm{KH}, \mathrm{Xu} \mathrm{H}, \mathrm{Wu} \mathrm{B}$. Gender differences in quality of life among community-dwelling older adults in low- and middle-income countries: results from the Study on global AGEing and adult health (SAGE). BMC Public Health. 2020;20(1):114. doi:10.1186/s12889020-8212-0

48. Lo CM, Lee PH. Prevalence and impacts of poor sleep on quality of life and associated factors of good sleepers in a sample of older Chinese adults. Health Qual Life Outcomes. 2012;10(1):72. doi:10.1186/1477-7525-10-72

49. Kempen GI, van Haastregt JC, McKee KJ, Delbaere K, Zijlstra GA. Socio-demographic, health-related and psychosocial correlates of fear of falling and avoidance of activity in community-living older persons who avoid activity due to fear of falling. BMC Public Health. 2009;9(1):170. doi:10.1186/1471-2458-9-170

50. Stenhagen M, Ekström H, Nordell E, Elmståhl S. Accidental falls, health-related quality of life and life satisfaction: a prospective study of the general elderly population. Arch Gerontol Geriatr. 2014;58 (1):95-100. doi:10.1016/j.archger.2013.07.006

51. Prata MG, Scheicher ME. Relationship between fear of falling and quality of life in older women fallers fear of falling and quality of life in older. MOJ Gerontol Ger. 2017;1(5):128-131.

52. Schoene D, Heller C, Aung YN, Sieber CC, Kemmler W, Freiberger E. A systematic review on the influence of fear of falling on quality of life in older people: is there a role for falls? Clin Interv Aging. 2019;14:701-719. doi:10.2147/CIA.S197857

53. Vennu V, Bindawas SM. Relationship between falls, knee osteoarthritis, and health-related quality of life: data from the Osteoarthritis Initiative Study. Clin Interv Aging. 2014;9:793-800. doi:10.2147/ CIA.S62207

54. Rodrigues I, Lima M, de Azevedo Barros M. Falls and health-related quality of life (SF-36) in elderly people-ISACAMP 2008. Health. 2013;5(12):49-57. doi:10.4236/health.2013.512A007

55. Farquhar M. Definitions of quality of life: a taxonomy. $J$ Adv Nurs. 1995;22(3):502-508. doi:10.1046/j.1365-2648.1995.22030502.x
Clinical Interventions in Aging

\section{Publish your work in this journal}

Clinical Interventions in Aging is an international, peer-reviewed journal focusing on evidence-based reports on the value or lack thereof of treatments intended to prevent or delay the onset of maladaptive correlates of aging in human beings. This journal is indexed on PubMed Central, MedLine, CAS, Scopus and the Elsevier

\section{Dovepress}

Bibliographic databases. The manuscript management system is completely online and includes a very quick and fair peer-review system, which is all easy to use. Visit http://www.dovepress.com/ testimonials.php to read real quotes from published authors. 